\title{
Frictionally Decaying Frontal Warm-Core Eddies
}

\author{
A. Rubino ${ }^{1, *}$, S. Dotsenko $^{2}$ \\ ${ }^{1}$ Dipartimento di Scienze Ambientali, Informatica e Statistica, Università Ca'Foscari, Venice, Italy \\ ${ }^{2}$ Marine Hydrophysical Institute of RAS, Sevastopol, Russian Federation \\ * rubino@unive.it
}

Purpose. The dynamics of nonstationary, nonlinear, axisymmetric, warm-core geophysical surface frontal vortices affected by Rayleigh friction is investigated semi-analytically using the nonlinear, nonstationary reduced-gravity shallow-water equations. The scope is to enlarge the number of known (semi)analytical solutions of nonstationary, nonlinear problems referring to geophysical problems and even to pave the way to their extension to broader geometries and/or velocity fields.

Methods and Results. The used method to obtain the solutions is based on the decomposition of the original equations in a part expressing their prescribed spatial structure, so that they can be transformed into ordinary differential equations depending on time only. Based on that analytical procedure, the solutions are then found numerically. In this frame, it is found that vortices characterized by linear distributions of their radial velocity and arbitrary structures of their section and azimuthal velocity can be described exactly by a set of nonstationary, nonlinear coupled ordinary differential equations. The first-order problem (i. e., that describing vortices characterized by a linear azimuthal velocity field and a quadratic section) consists of a system of 4 differential equations, and each further order introduces in the system three additional ordinary differential equations and two algebraic equations. In order to illustrate the behavior of the nonstationary decaying vortices and to put them in the context of observed dynamics in the World Ocean, the system's solution for the first-order and for the second-order problem is then obtained numerically using a Runge-Kutta method. The solutions demonstrate that inertial oscillations and an exponential attenuation dominate the vortex dynamics: expansions and shallowings, contractions and deepenings alternate during an exact inertial period while the vortex decays. The dependence of the vortex dissipation rate on its initial radius is found to be non-monotonic: it is higher for small and large radii. The possibility of solving (semi)analytically complex systems of differential equations representing observed physical phenomena is rare and very valuable. Conclusions. Our analysis adds realism to previous theoretical investigations on mesoscale vortices, represents an ideal tool for testing the accuracy of numerical models in simulating nonlinear, nonstationary frictional frontal phenomena in a rotating ocean, and paves the way to further extensions of (semi-) analytical solutions of hydrodynamical geophysical problems to more arbitrary forms and more complex density stratifications.

Keywords: geophysical vortices, analytical solutions, ocean mesoscale.

Acknowledgements: a previous version of this paper can be found in the arXiv repository (https://arxiv.org/abs/1607.01665).

For citation: Rubino, A. and Dotsenko, S., 2020. Frictionally Decaying Frontal Warm-Core Eddies. Physical Oceanography, [e-journal] 27(4), pp. 442-453. doi:10.22449/1573-160X-2020-4-442-453

DOI: $10.22449 / 1573-160 X-2020-4-442-453$

(C) A. Rubino, S. Dotsenko, 2020

(C) Physical Oceanography, 2020

\section{INTRODUCTION}

Geophysical frontal vortices are frequently observed in the ocean and they have attracted the interest of a large number of investigators (see, e. g., [1, 2] for a review of particularly significant results). Particularly, they are believed to play a fundamental role in different, important oceanic phenomena like, e. g., those related to the transfer of physical, chemical, and biological properties across frontal regions (see, e. g., [3-9]), to the formation and transformation of water masses (see, e. g., $[10,11]$ ), and to the 
downward propagation of wind generated near-inertial waves [12, 13]. This extraordinarily large relevance explains why, in the last decades, oceanic frontal vortices have been deeply investigated experimentally, analytically and numerically (see, e. g., [1, 14-21]). Note that the geophysical relevance of those features involves not only the near-surface ocean, but its interior and abyssal layers as well.

A prominent role in the theoretical investigation of geophysical frontal vortices has been played by the nonlinear, reduced gravity shallow water equations [18, 20, 22-26].

Although these equations do not allow for the development of baroclinic instabilities or for the radiation of energy toward the interior ocean via internal waves propagation, their use enables to explain different characteristics of observed frontal vortices [18, 20, 22-26]. Moreover, the possibility of expressing analytically the evolution of a broad class of geophysical frontal vortices using these equations represents a valuable way for testing the accuracy of numerical models for non-stationary, nonlinear, frontal phenomena of geophysical relevance [18, 19, 22, 27].

Fundamentally, in the case of circular symmetry, the features emerging as analytical solutions in the reduced-gravity frame are characterized by inertial oscillations affecting both radial and tangential velocities and vortex's depth: The vortices contract and expand during an exact inertial period [22, 25]. Note that, in these analytical solutions, the vortex's radial velocity field is constrained to be a linear function of the vortex's radius, while the azimuthal velocity field and the vortex's section can show a more complex shape $[25,26]$. A More complex behavior is also possible for frontal vortices characterized by elliptic sections: In this case subinertial and superinertial oscillations emerge as a part of the corresponding analytical solution (see [22]).

On the (unavoidably obscure and hence also profoundly fascinating) way of searching for analytical solutions of systems of unsteady, nonlinear coupled partial differential equations the fundamental step consists in their reduction to a system of ordinary differential equations. A possibility is to prescribe the spatial characteristics of (part of) the system, hence reducing the problem to the search of the temporal behavior alone Has this step been performed, so different methods can be attempted to solve analytically the obtained system. But, even if an analytical solution cannot be found, once the reduction has been performed, numerical methods often allow then for an extremely accurate simulation of the involved dynamics, as the originally multidimensional problem has been reduced to a monodimensional one.

In the case of frontal mesoscale vortices of the ocean, one has also to note that, among the different scales characterizing their temporal variability (from the superinertial ones affecting the swirl velocity of elongated elliptical anticyclones to the exactly inertial one typical of the circular pulson and to the super inertial subinertial modes emerging in warm-core eddies on a $\beta$-plane, see, e. g., $[20,24])$ that associated with their frictional decay plays a particular role, because it contributes to determine the fate of these mesoscale features: In the case of warm-core rings, for instance, their remarkable longevity enables disintegration in coastal areas and/or re-absorption by the parent current to fundamentally contribute to their dissipation/variability [28-30]. Note that a similar longevity can be observed for the corresponding features of the interior ocean [5, 31]. 
In the present investigation, the dynamics of nonstationary, nonlinear, axisymmetric, warm-core geophysical surface frontal vortices affected by Rayleigh friction is analyzed semi-analytically using the nonlinear, nonstationary reducedgravity shallow-water equations. In order to illustrate the behavior of the nonstationary decaying vortices and to put them in the context of observed dynamics in the World Ocean, the system's solution for the first-order and for the second-order problem is then obtained numerically using a Runge-Kutta method. The solutions demonstrate that inertial oscillations and an exponential attenuation dominate the vortex dynamics: Expansions and shallowings, contractions and deepenings alternate during an exact inertial period while the vortex decays. The dependence of the vortex dissipation rate on its initial radius is found to be non-monotonic: It is higher for small and large radii. Our analysis adds realism to previous theoretical investigations on mesoscale vortices, represents an ideal tool for testing the accuracy of numerical models in simulating nonlinear, nonstationary frictional frontal phenomena in a rotating ocean, and paves the way to further extensions of (semi-) analytical solutions of hydrodynamical geophysical problems to more arbitrary forms and more complex density stratifications.

The paper is organized as follows: In the next section the mathematical model is illustrated and its reduction to a system of ordinary differential equations is presented. Numerical solutions obtained using a Runge-Kutta methods are discussed in section 3. Finally, the obtained results are discussed und conclusions drawn in section 4 .

\section{THE MATEMATICAL MODEL}

In the frame of the non-stationary, nonlinear reduced-gravity equations on an $f$-plane we consider the frictional (Rayleigh) dynamics of a circular, frontal warmcore eddy: A lens of light water of density $\rho$ lies on the top of a heavier, infinitely deep quiescient ocean of density $\rho^{*}$ (Fig. 1). Assuming circular symmetry, the motion of the active layer can be expressed as follow in cylindrical coordinates $(r, z, \varphi)$ :

$$
\begin{gathered}
\frac{\partial u}{\partial t}+u \frac{\partial u}{\partial r}-\frac{v^{2}}{r}-f v=-g^{\prime} \frac{\partial h}{\partial r}-s u \\
\frac{\partial v}{\partial t}+u \frac{\partial v}{\partial r}+\frac{u v}{r}+f u=-s v \\
\frac{\partial h}{\partial t}+\frac{1}{r} \frac{\partial(r u h)}{\partial r}=0
\end{gathered}
$$

In the expressions above, $t$ is the time, $\{u ; v\}(r, t)$ are, respectively, the radial and the azimuthal projections of the horizontal velocity, $h(r, t)$ is the vortex thickness, $g^{\prime}=g\left(1-\rho / \rho^{*}\right)$ the reduced gravity (where $g$ represents the acceleration of gravity), while $f$ is the (constant) Coriolis parameter, and $s$ the (constant) friction coefficient.

Any solution of the system (1) - (3) has to satisfy the condition

$$
h\left(r_{0}, t\right)=0
$$




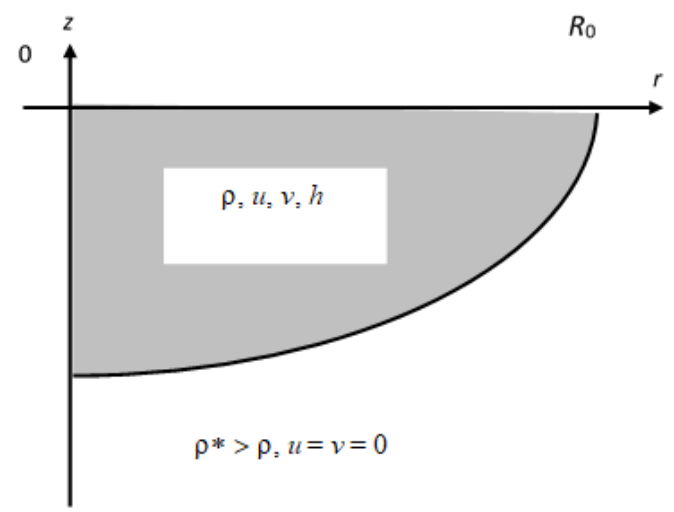

F i g. 1. Radial section of the considered vortices

on the movable surface circular boundary of the vortex (its surface rim) located at $r=r_{0}$ (see Fig. 1).

Reduction of model to a system of ordinary differential equations. Let us now assume that velocity fields and vortex thickness be characterized by the following horizontal structures:

$$
u=A(t) r, \quad v=\sum_{i=1}^{N} B_{i}(t) r^{2 i-1}, \quad h=\sum_{j=0}^{2 N-1} C_{j}(t) r^{2 j},
$$

where $A, B_{i}$ and $C_{j}$ are functions of time only, and $N \geq 1$. Note that the condition that the thickness $h$ of the vortex be positive and satisfy (5) implies

$$
\sum_{j=1}^{2 N-1} C_{j}(0)>0
$$

A substitution of (5) into (1) - (3) yields the following system of nonstationary, nonlinear, coupled, ordinary differential equations (ODEs) and algebraic equations in the unknown functions $A, B_{i}$ and $C_{i}$ :

$$
\begin{gathered}
\delta_{i 1} \frac{d A}{d t}+\delta_{i 1} A^{2}-\sum_{j=1}^{i} B_{j} B_{i-j+1}-f B_{1}+2 i g C_{i}+s A_{i}=0 \quad(i=1, \ldots, 2 N-1), \\
\frac{d B_{i}}{d t}+2 i A B_{i}+\delta_{i 1} f A+s B_{i}=0 \quad(i=1, \ldots, N), \\
\frac{d C_{i}}{d t}+2(i+1) A C_{i}=0 \quad(i=0, \ldots, 2 N-1),
\end{gathered}
$$

where $\delta_{i l}$ is the Kronecker delta, and $B_{i}=0$ for $i>N$. The initial conditions for the coefficients $A, B_{i}$ and $C_{i}$ are:

$$
A(0)=A_{0}, \quad B_{i}(0)=B_{i 0}, \quad C_{i}(0)=C_{i 0} .
$$


First-order reduction $(\boldsymbol{N}=\mathbf{1})$. In order to discuss fundamental characteristics of the dynamics of frictional vortices described above, in the following we will focus on their first-order and second-order expressions.

Let us consider the first-order expression of the vortex fields expressed in (5) (7):

$$
u=A(t) r, \quad v=B_{1}(t) r, \quad h=C_{0}(t)+C_{1}(t) r^{2},
$$

with $A, B_{1}, C_{0}$ and $C_{1}$ unknown functions of time only. The requirement that the vortex has positive thickness $h$ and the condition (4) yield:

$$
C_{0}(t)>0, \quad C_{1}(t)<0 .
$$

Substituting the expressions (9) into (1) - (3) and equating the coefficients of equal powers in $r$ leads to a system of four ODEs in the variables $A, B_{1}, C_{0}$ and $C_{1}$ :

$$
\begin{gathered}
\frac{d A}{d t}+A^{2}-B_{1}^{2}-f B_{1}+2 g^{\prime} C_{1}+s A=0, \\
\frac{d B_{1}}{d t}+2 A B_{1}+f A+s B_{1}=0, \\
\frac{d C_{0}}{d t}+2 A C_{0}=0, \\
\frac{d C_{1}}{d t}+4 A C_{1}=0 .
\end{gathered}
$$

The system of equations (12) - (15) must be supplemented with the following initial conditions:

$$
A(0)=A_{0}, \quad B_{1}(0)=B_{10}, \quad C_{0}(0)=C_{00}>0, \quad C_{1}(0)=C_{10}<0 .
$$

Second-order reduction ( $\mathbf{N}=\mathbf{2})$. Let us now pass to the second-order expression of the vortex fields:

$$
u=A(t) r, \quad v=B_{1}(t) r+B_{2}(t) r^{3}, \quad h=C_{0}(t)+C_{1}(t) r^{2}+C_{2}(t) r^{4}+C_{3}(t) r^{6},
$$

with $A, B_{1,2}$ and $C_{0,1,2,3}$ unknown functions of time only. Substituting the expressions (17) into (1) - (3) and equating the coefficients of equal powers in $r$ leads to a system of seven ODEs and two algebraic equations in the variables $A, B_{1,2}, C_{0,1,2,3}$ :

$$
\begin{gathered}
\frac{d A}{d t}+A^{2}-B_{1}^{2}-f B_{1}+2 g^{\prime} C_{1}+s A=0 \\
2 B_{1} B_{2}+f B_{2}-4 g^{\prime} C_{2}=0 \\
B_{2}^{2}-6 g^{\prime} C_{3}=0 \\
\frac{d B_{1}}{d t}+2 A B_{1}+f A+s B_{2}=0 \\
\frac{d B_{2}}{d t}+4 A B_{2}+s B_{2}=0
\end{gathered}
$$




$$
\begin{aligned}
& \frac{d C_{0}}{d t}+2 A C_{0}=0, \\
& \frac{d C_{1}}{d t}+4 A C_{1}=0, \\
& \frac{d C_{2}}{d t}+6 A C_{2}=0, \\
& \frac{d C_{3}}{d t}+8 A C_{3}=0 .
\end{aligned}
$$

Analytical solution of the first-order problem without dissipation. To illustrate the general characteristics of the dynamics of the inviscid $(s=0)$ solutions of (6) - (8) (see, e. g., [24, 25]) we will shortly summarize the properties of the firstorder analytical solution. In this case, indeed, one finds:

$$
A=\frac{1}{2} \gamma f \Psi \cos \Phi, \quad B_{1}=-\frac{1}{2} f+l \Psi, \quad C_{0}=c \Psi, \quad C_{1}=-\frac{c}{R_{0}^{2}} \Psi^{2},
$$

where $R_{0}$ is the initial vortex radius,

$$
\Psi=\frac{1}{1+\gamma \sin \Phi}, \Phi=f t+\mathrm{f} \text {, and } \quad l=\frac{1}{2} f \sqrt{1-\gamma^{2}-\frac{8 g^{\prime} c}{f^{2} R_{0}^{2}}} .
$$

Here $\in[0,1], \varphi$, and $c>0$ are constants. The solution (27) describes a pulsating vortex: Expansions and shallowings, contractions and deepenings alternate during an exact inertial period [24, 25]. Note that the pulsation amplitude is determined by the value of $\gamma$. In the numerical experiments of the following section this solution will be used to define the (ageostrophic) initial conditions for calculating of the vortex oscillations in the frictional case.

The kinetic, potential, and total energy, $E_{\mathrm{kin}}, E_{\mathrm{pot}}$, and $E_{\mathrm{tot}}$ of the vortex, whose structure is described by (6) - (8) can be expressed as:

$$
E_{\text {pot }}=\frac{1}{2} \rho g^{\prime} \iint_{S} h^{2} d x d y, \quad E_{\text {kin }}=\frac{1}{2} \rho \iint_{S} h\left(u^{2}+v^{2}\right) d x d y,
$$

where $S$ corresponds to the time-dependent vortex surface.

\section{NUMERICAL SOLUTIONS}

First-order solutions. The system of equations (12) - (15) with the appropriate initial conditions (16) was solved numerically using a Runge-Kutta method of the fourth-order.

In order to select realistic values for the solution we refer to the detailed statistics of the Gulf Stream rings observed between 1974 and 1983 which can be found in [32].

An appropriate value for the friction coefficient $s$ in Eqs. (1), (2) can be selected to reflect observed lifetimes of geophysical oceanic vortices. Assuming an exponential decay of the vortex velocity field, $t_{\text {life }}$ will represent the vortex $e$-folding time, and hence $s=1 / t_{\text {life. }}$ Typical observed values for Gulf Stream rings are [32]: initial radius $R_{0}=75 \mathrm{~km}, t_{\text {life }}=130$ days, latitude $=38^{\circ} \mathrm{N}$ and initial depth $h_{0}=500 \mathrm{~m}$. Therefore, the corresponding friction coefficient $s=1 / t_{\text {life }}=8,903 \cdot 10^{-8} \mathrm{~s}^{-1}$. 
In our evaluations we set moreover $g^{\prime}=0,01 \mathrm{~m} \cdot \mathrm{s}^{-2}$ for the density difference between vortex and ambient water, and $\varphi=0$.

The temporal evolution of a first-order frictional vortex is shown in Fig. 2: in particular, depicted are the radial velocity $U=A(t) r_{0}(t)$ and the azimuthal velocity $V=B_{1}(t) r_{0}(t)$ at the (temporally varying) vortex rim $r_{0}(t)$, as well as the maximum vortex thickness $H=C_{0}(t)$ at its center. Exact inertial oscillations clearly emerge: as in the inviscid case, expansions and shallowings, contractions and deepenings alternate during an exact inertial period [24, 25], while both velocity amplitudes and maximum thickness decrease and radius increases as time elapses due to the action of the Rayleigh friction.
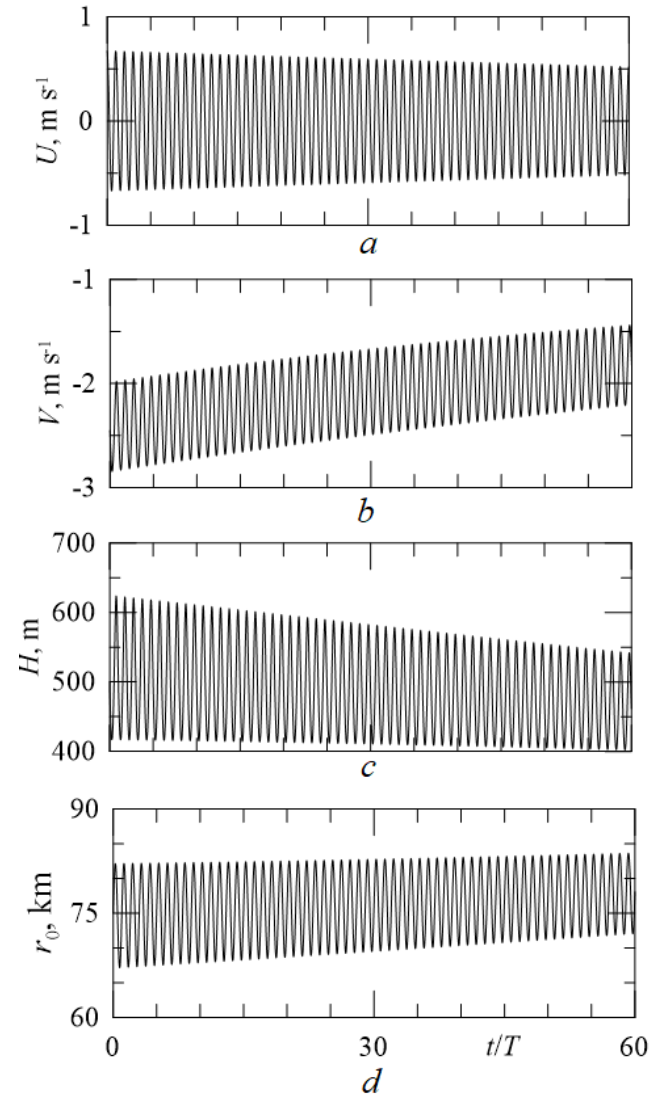

F i g. 2. Inertial oscillations and attenuation of radial $(a)$ and azimuthal $(b)$ components of the horizontal velocity, maximum depth $(c)$ and radius (d) of the vortex. The initial radius of vortex is $R_{0}=75 \mathrm{~km}$, the amplitude oscillation parameter is $\gamma=0.2$. $T$ represents the inertial period $(T=19.49 \mathrm{~h})$

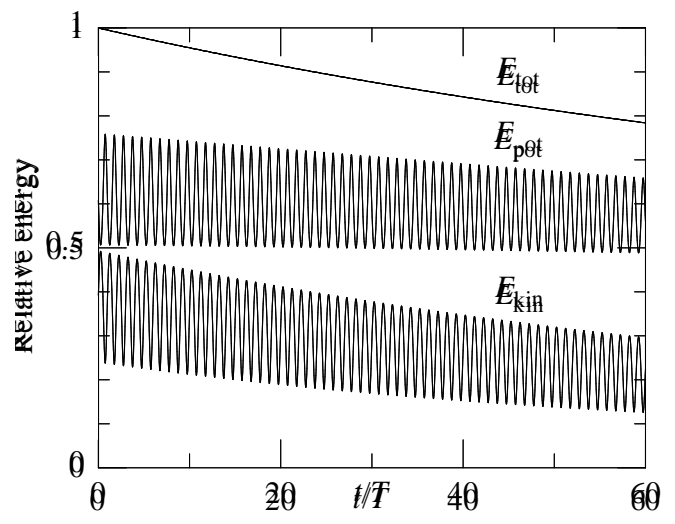

F i g. 3. Temporal evolution of the kinetic $\left(E_{\text {kin }}\right)$, potential $\left(E_{\mathrm{pot}}\right)$, and total $\left(E_{\mathrm{tot}}\right)$ energy of the vortex. The initial radius of the vortex is $R_{0}=$ $=75 \mathrm{~km}$, the amplitude oscillation parameter is $\gamma=0.2$

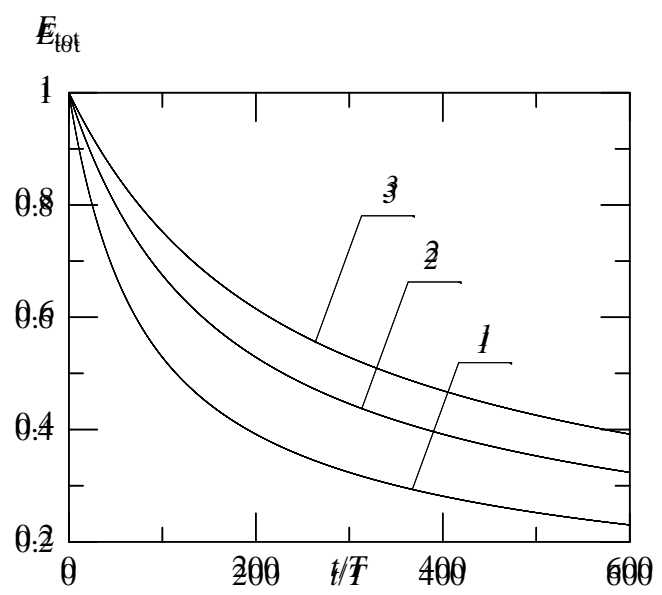

F i g. 4. Temporal evolution of the vortex total energy $\left(E_{\text {tot }}\right)$ for different durations vortex longevities: $1-t_{\text {life }}=60$ days; $2-t_{\text {life }}=120$ days; $3-t_{\text {life }}$ $=180$ days. The initial vortex radius is $R_{0}=75 \mathrm{~km}$, the amplitude oscillation parameter is $\gamma=0.2$ 
Frictional decay is also evident in the evolution of the vortex integral energy characteristics (Fig. 3). As in the inviscid case, vortex kinetic and potential energy show exact inertial anti-phase oscillations, however, they also decay in time together with the vortex total energy which monotonously decreases.

In Fig. 4 the vortex total energy decay is depicted as a function of different vortex frictional coefficients (or, equivalently, as a function of different vortex longevities). Larger frictions produce faster energy decays, the latter decreasing with increasing coefficients. Larger oscillations (expressed by the magnitude of the parameter $\gamma$ ) lead to faster energy dissipation (Fig. 5). In Fig. 6 the temporal evolution of the total energy is depicted as a function of the initial vortex radius. The dissipation rate of is the largest for initially small and large features, and smaller for intermediate ones.

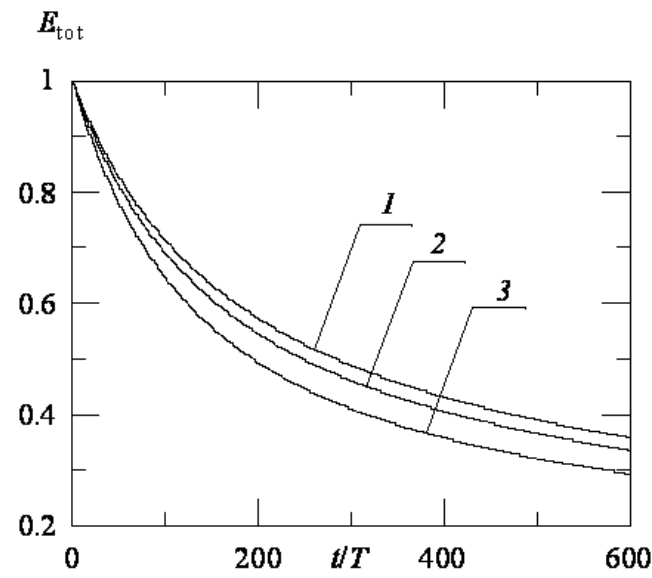

F i g. 5. Temporal evolution of the vortex total energy $\left(E_{\text {tot }}\right)$ for different values of the amplitude oscillation parameter $\gamma: 1-\gamma=0.1 ; 2-\gamma=0.2$; $3-\gamma=0.3$. The initial vortex radius is $R_{0}=$ $=75 \mathrm{~km}$, the friction coefficient is $s=8.90310^{-8} \mathrm{~s}^{-1}$

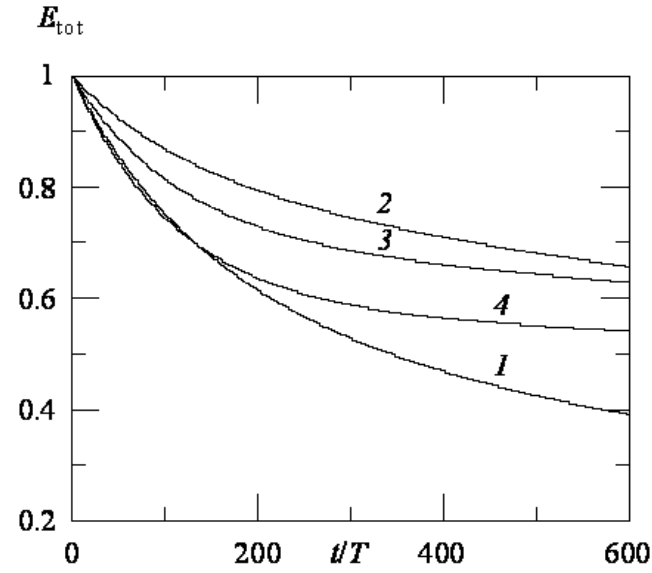

F i g. 6. Temporal evolution of the vortex total energy $\left(E_{\text {tot }}\right)$ for different values of the initial vortex radius: $1-R_{0}=75 \mathrm{~km} ; 2-R_{0}=150 \mathrm{~km} ; 3-$ $R_{0}=225 \mathrm{~km} ; 4: R_{0}=300 \mathrm{~km}$. The amplitude oscillation parameter is $\gamma=0.2$, the friction coefficient is $s=8.903 \cdot 10^{-8} \mathrm{~s}^{-1}$

Higher-order solutions. As an example of the frictional vortex evolution for a high-order pulson we now present results referring to the second-order problem, which is described by the system (18) - (26). Like in the previous case, the solutions have been computed numerically using a fourth-order Runge-Kutta algorithm.

The evolution of the vortex velocity fields and radius closely resembles that elucidated for the fist-order solution (Fig. 7). As time elapses, the amplitudes of the oscillations (which are still exactly inertial) decrease and the radius increases as time elapses, due to the action of the Rayleigh friction.

Associated to this behavior we note a more complex evolution of the different fields. As indicated previously, in the inviscid as well as in the frictional solutions the radial velocity $u$ has to be a linear function of radius for each order and frictional coefficient in order for the problem to be reduced to a system of ordinary differential equations. The influence of friction, hence, cannot alter the shape of the radial velocity horizontal distribution: Instead, it leads to a monotonic decrease of 
the radial velocity amplitude with increasing values of the frictional parameter $s$. In the second-order problem considered, the inviscid tangential velocity $v$ used as initial condition for the frictional simulations largely deviates from the linear one characterizing the first-order problem. In this case (not shown), it reaches its maximum well within the vortex body and then decreases toward the periphery. Note that such kind of distributions has been found as being the one typically emerging in the first evolutionary stage of an impulsive vortex generation obtained in tank experiments devoted at investigating geophysical frontal vortices (see [18]). Friction induces a tendency toward a decreasing amplitude and nonlinearity of the tangential velocity distribution. The region of maximum values experiences a shift toward the vortex periphery as the friction coefficient $s$ increases. Accordingly, the deviation in the form of the vortex section from a parabolic one also decreases as the friction increases.
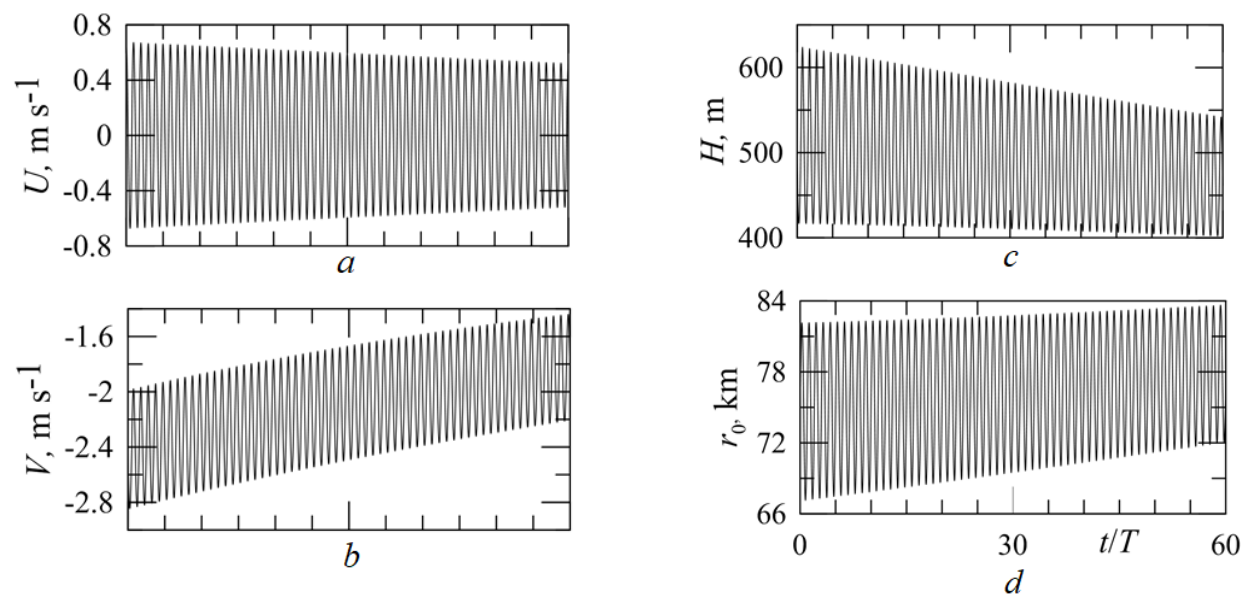

F i g. 7. Inertial attenuating oscillations of radial $(a)$ and azimuthal $(b)$ components of the horizontal velocity, maximum depth $(c)$ and radius $(d)$ of a second-order vortex solution. The vortex is characterized initially by a radius $R_{0}=75 \mathrm{~km}$, a thickness $h_{0}=500 \mathrm{~m}$, and an amplitude oscillation parameter $\gamma=0,2$. The friction coefficient corresponds to a $t_{\text {life }}=130$ days, i.e., $s=1 / t_{\text {life }}=8,903 \cdot 10^{-8} \mathrm{~s}^{-1}$

\section{DISCUSSION AND CONCLUSIONS}

In the present paper we have analyzed aspects of the dynamics of nonstationary, nonlinear, axisymmetric, warm-core geophysical surface frontal vortices affected by Rayleigh friction in the frame of the nonlinear, nonstationary reducedgravity shallow-water equations. In this frame, we have shown that, in the case of circular features characterized by radial velocities which are linear functions of the vortex radius, it is possible to reduce the problem to a set of ordinary differential equations like in the case of the pulson described by [25]. This step, which is performed by prescribing the spatial structure of the investigated eddy and hence reducing the vortex variability to the temporal one alone, is the fundamental one in the (unavoidably obscure) search of exact analytical solutions of complex problems expressed in terms of nonstationary, nonlinear, coupled partial differential equations depending on space and time. In the present case, however, it seems that obtaining exact analytical solutions is not a straightforward task. Hence, we concentrated on numerical solutions using a fourth-order Runge-Kutta method. These so- 
lutions, however, can be believed to represent an excellent approximation to the exact ones, as the problem has been reduced to a monodimensional one and hence the method allows for a very high accuracy.

In order to illustrate the behavior of the decaying vortices, the system's solutions for the first-order and for the second-order problem have been considered. For both cases, inertial oscillations and an exponential attenuation dominate the vortex dynamics: Expansions and shallowings, contractions and deepenings alternate during an exact inertial period while the vortex decays. The dependence of the vortex dissipation rate on its initial radius is found to be non-monotonic: It is higher for small and large radii. For realistic values of the friction coefficient we obtain a relatively high longevity of the simulated features, which is consistent with many observations indicating as long-living both the features of the near-surface and those of the interior ocean. Obviously, our study in the frame of the reducedgravity theory does not allow for many of the interactions with the ambient ocean and the atmosphere which are known to be able to exert an influence on the stability, attenuation, but also reemergence of frontal features in the real ocean. [9] for instance, using a hierarchy of coupled realistic numerical models studied the persistency of a convectively generated frontal vortex in the Greenland Sea: here the interaction with the atmosphere can contribute to the preservation of the feature at the ocean near-surface or to its transformation into an intermediate one, which, possibly can reemerge throug convective activity.

Nevertheless, our analysis adds realism to previous semi-analytical investigations on mesoscale vortices, which were limited to the inviscid case and, most of them, to the first-order solution of the pulson family. It also represents an ideal tool for testing the accuracy of three-dimensional numerical models in simulating realistic geophysical problems, as it refers to nonlinear, nonstationary frictional frontal dynamics in a rotating ocean. Moreover, it paves the way to further extensions of (semi-) analytical solutions of hydrodynamical geophysical problems to more arbitrary forms and more complex density stratifications.

\section{REFERENCES}

1. McWilliams, J.C., 1985. Submesoscale, Coherent Vortices in the Ocean. Reviews of Geophysics, 23(2), pp. 165-182. https://doi.org/10.1029/RG023i002p00165

2. Olson, D.B., 1991. Rings in the Ocean. Annual Review of Earth and Planetary Sciences, 19, pp. 283-311. https://doi.org/10.1146/annurev.ea.19.050191.001435

3. Saunders, P.M., 1971. Anticyclonic Eddies Formed from Shoreward Meanders of the Gulf Stream. Deep Sea Research and Oceanographic Abstracts, 18(12), pp. 1207-1219. https://doi.org/10.1016/0011-7471(71)90027-1

4. Cheney, R.E., Gemmill, W.H., Shank, M.K., Richardson, P.L. and Webb, D., 1976. Tracking a Gulf Stream Ring with SOFAR Floats. Journal of Physical Oceanography, 6(5), pp. 741749. https://doi.org/10.1175/1520-0485(1976)006<0741:TAGSRW>2.0.CO;2

5. Armi, L. and Zenk, W., 1984. Large Lenses of Highly Saline Mediterranean Water. Journal of Physical Oceanography, 14(10), pp. 1560-1576. https://doi.org/10.1175/15200485(1984)014<1560:LLOHSM>2.0.CO;2

6. Joyce, T.M., 1984. Velocity and Hydrographic Structure of a Gulf Stream Warm-Core Ring. Journal of Physical Oceanography, 14(5), pp. 936-947. https://doi.org/10.1175/15200485(1984)014<0936:VAHSOA>2.0.CO;2 
7. Olson, D.B., Schmitt, R.W., Kennelly, M. and Joyce, T.M., 1985. A Two-Layer Diagnostic Model of the Long-Term Physical Evolution of Warm-Core Ring 82B. Journal of Geophysical Research: Oceans, 90(C5), pp. 8813-8822. https://doi.org/10.1029/JC090iC05p08813

8. Dengler, M., Schott, F., Eden, C., Brandt, P., Fischer, J. and Zantopp, R.J., 2004. Break-up of the Atlantic Deep Western Boundary Current into Eddies at $8^{\circ}$ S. Nature, 432(7020), pp. 1018-1020. https://doi.org/10.1038/nature03134

9. Rubino, A., Androssov, A. and Dotsenko, S., 2007. Intrinsic Dynamics and Long-Term Evolution of a Convectively Generated Oceanic Vortex in the Greenland Sea. Geophysical Research Letters, 34(16), L16607. doi:10.1029/2007GL030634

10. Gascard, J.-C., Watson, A.J., Messias, M.-J., Olson, K.A., Johannessen, T. and Simonsen, K., 2002. Long-Lived Vortices as a Mode of Deep Ventilation in the Greenland Sea. Nature, 416(6880), pp. 525-527. https://doi.org/10.1038/416525a

11. Budéus, G., Cisewski, B., Ronski, S., Dietrich, D. and Weitere, M., 2004. Structure and Effects of a Long Lived Vortex in the Greenland Sea. Geophysical Research Letters, 31(5), L05304. doi:10.1029/2003GL017983

12. Lee, D.-K. and Niiler, P.P., 1998. The Inertial Chimney: The Near-Inertial Energy Drainage from the Ocean Surface to the Deep Layer. Journal of Geophysical Research: Oceans, 103(C4), pp. 7579-7591. https://doi.org/10.1029/97JC03200

13. Zhai, X., Greatbatch, R.J. and Eden, C., 2007. Spreading of Near-Inertial Energy in a 1/12 ${ }^{\circ}$ Model of the North Atlantic Ocean. Geophysical Research Letters, 34(10), L10609. doi:10.1029/2007GL029895

14. Csanady, G.T., 1979. The Birth and Death of a Warm Core Ring. Journal of Geophysical Research: Oceans, 84(C2), pp. 777-780. https://doi.org/10.1029/JC084iC02p00777

15. Gill, A.E., 1981. Homogeneous Intrusions in a Rotating Stratified Fluid. Journal of Fluid Mechanics, 103, pp. 275-295. https://doi.org/10.1017/S0022112081001341

16. Nof, D., 1983. On the Migration of Isolated Eddies with Application to Gulf Stream Rings. Journal of Marine Research, 41(3), pp. 399-425. https://doi.org/10.1357/002224083788519687

17. McWilliams, J.C., 1988. Vortex Generation through Balanced Adjustment. Journal of Physical Oceanography, 18(8), pp. 1178-1192. https://doi.org/10.1175/1520-0485(1988)018<1178: VGTBA $>2.0 . \mathrm{CO} ; 2$

18. Rubino, A. and Brandt, P., 2003. Warm-Core Eddies Studied by Laboratory Experiments and Numerical Modeling. Journal of Physical Oceanography, 33(2), pp. 431-435. https://doi.org/10.1175/1520-0485(2003)033<0431:WCESBL >2.0.CO;2

19. Rubino, A., Hessner, K. and Brandt, P., 2002. Decay of Stable Warm-Core Eddies in a Layered Frontal Model. Journal of Physical Oceanography, 32(1), pp. 188-201. https://doi.org/10.1175/1520-0485(2002)032<0188:DOSWCE >2.0.CO;2

20. Rubino, A., Dotsenko, S. and Brandt, P., 2009. Nonstationary Westward Translation of Nonlinear Frontal Warm-Core Eddies. Journal of Physical Oceanography, 39(6), pp. 1486-1494. https://doi.org/10.1175/2008JPO4089.1

21. Rubino, A., Gačić, M., Bensi, M., Kovačević, V., Malačič, V., Menna, M., Negretti, M.E., Sommeria, J., Zanchettin, D., Barreto, R.V., Ursella, L., Cardin, V., Civitarese, G., Orlić, M., Petelin, B. and Siena, G., 2020. Experimental Evidence of Long-Term Oceanic Circulation Reversals without Wind Influence in the North Ionian Sea. Scientific Reports, 10, 1905. https://doi.org/10.1038/s41598-020-57862-6

22. Cushman-Roisin, B., 1987. Exact Analytical Solutions for Elliptical Vortices of the Shallow-Water Equations. Tellus A, 39A(3), pp. 235-244. https://doi.org/10.1111/j.16000870.1987.tb00304.x

23. Cushman-Roisin, B. and Merchant-Both, S., 1995. Elliptical Warm-Core Rings in a TwoLayer Ocean with Ambient Shear. Journal of Physical Oceanography, 25(9), pp. 2011-2024. https://doi.org/10.1175/1520-0485(1995)025<2011:EWCRIA>2.0.CO;2 
24. Cushman-Roisin, B., Heil, W.H. and Nof, D., 1985. Oscillations and Rotations of Elliptical Warm-Core Rings. Journal of Geophysical Research: Oceans, 90(C6), 11756- 11764. https://doi.org/10.1029/JC090iC06p11756

25. Rubino, A., Brandt, P. and Hessner, K., 1998. Analytical Solutions for Circular Eddies of the Reduced-Gravity, Shallow-Water Equations. Journal of Physical Oceanography, 28(5), pp. 999-1002. https://doi.org/10.1175/1520-0485(1998)028<0999:ASFCEO>2.0.CO;2

26. Rubino, A. and Dotsenko, S., 2006. The Stratified Pulson. Journal of Physical Oceanography, 36(4), pp. 711-719. https://doi.org/10.1175/JPO2863.1

27. Cushman-Roisin, B., 1986. Frontal Geostrophic Dynamics. Journal of Physical Oceanography, 16(1), pp. 132-143. https://doi.org/10.1175/1520-0485(1986)016<0132:FGD>2.0.CO;2

28. Flierl, G.R. and Mied, R.P., 1985. Frictionally Induced Circulations and Spin Down of a Warm-Core Ring. Journal of Geophysical Research: Oceans, 90(C5), pp. 8917-8927. https://doi.org/10.1029/JC090iC05p08917

29. Tomosada, A., 1986. Generation and Decay of Kuroshio Warm-Core Rings. Deep Sea Research Part A. Oceanographic Research Papers, 33(11-12), pp. 1475-1486. https://doi.org/10.1016/0198-0149(86)90063-4

30. Itoh, S., Shimizu, Y., Ito, S. and Yasuda, I., 2001: Evolution and Decay of a Warm-Core Ring within the Western Subarctic Gyre of the North Pacific, as Observed by Profiling Floats. Journal of Oceanography, 67(3), pp. 281-293. https://doi.org/10.1007/s10872-011-0027-2

31. Armi, L., Hebert, D., Oakey, N., Price, J.F., Richardson, P.L., Rossby, H.T. and Ruddick, B., 1989. Two Years in the Life of a Mediterranean Salt Lens. Journal of Physical Oceanography, 19(3), pp. 354-370. https://doi.org/10.1175/1520-0485(1989)019<0354:TYITLO>2.0.CO;2

32. Brown, O.B., Cornillon, P.C., Emmerson, S.R. and Carle, H.M., 1986. Gulf Stream Warm Rings: A Statistical Study of Their Behavior. Deep Sea Research Part A. Oceanographic Research Papers, 33(11-12), pp. 1459-1473. https://doi.org/10.1016/0198-0149(86)90062-2

About the authors:

Angelo Rubino - Professor, Department of Environmental Sciences, Informatics and Statistics, Ca' Foscari University of Venice (155, Via Torino, Mestre (Venice), 30175, Italy), Doctor of Oceanography, ORCID ID: 0000-0003-3857-4811, Scopus Author ID: 56277543800

Sergey F. Dotsenko - Senior Research Associate, Marine Hydrophysical Institute of RAS (2 Kapitanskaya St., Sevastopol, 299011, Russian Federation), Ph. D. (Phys.-Math.), Scopus Author ID: 7006290265

Contribution of the co-authors:

Angelo Rubino - conceiving the paper, discussion of the results, writing the paper

Sergey F. Dotsenko - conceiving the paper, carrying out the calculations, discussion of the results, writing the paper 